Rev. salud pública. 14 (1): 116-128, 2012

\title{
Problemas y decisiones al final de la vida en pacientes con enfermedad en etapa terminal
}

\author{
Terminally-ill patients' end of life problems and related decisions
}

María I. Sarmiento-Medina ${ }^{1}$, Sandra L. Vargas-Cruz ${ }^{1}$, Claudia M. Velásquez-Jiménez² y Margarita Sierra de Jaramillo ${ }^{1}$

1 Universidad El Bosque. Bogotá, Colombia. sarmientomaria@unbosque.edu.co, vargassandra@ unbosque.edu.co, sierramargarita@unbosque.edu.co

2 Fundación Universitaria Sanitas. Bogotá, Colombia. cmvelasquez@unisanitas.edu.co

Recibido 14 Marzo 2011/ Enviado para Modificación 5 Diciembre 2011/Aceptado 31 Enero 2012

\section{RESUMEN}

Objetivos Describir preferencias en las decisiones al final de la vida en pacientes y familiares, problemas que subyacen y motivos que los llevan a solicitar apoyo. Métodos Estudio descriptivo, exploratorio de fuentes secundarias de una ONG que brinda apoyo a los pacientes al final de la vida.

Resultados La opción más frecuente fue la muerte digna en $43 \%$ seguida de la eutanasia en un $12 \%$. Un $22 \%$ de las personas solicitaban orientación sobre aspectos éticos y legales y además referentes a la atención adecuada para enfermos terminales. Las patologías que ocuparon el $75 \%$ de los diagnósticos fueron Enfermedad Crónica Degenerativa, Cáncer y Estado de Coma. La agudización de síntomas fue el principal motivo por el cual solicitaron apoyo y el dolor incontrolable llevó con más frecuencia a la opción por la eutanasia. Sólo 14 \% de los pacientes habían formalizado sus voluntades anticipadas respecto al final de la vida. La opción de muerte digna se relacionó con el rechazo a medidas fútiles y al encarnizamiento terapéutico. La eutanasia se percibió como acción intencional y autónoma de poner fin al sufrimiento. La familia juega un papel esencial en la toma de decisiones.

Conclusiones Las decisiones al final de la vida son motivadas por la percepción de unas condiciones no dignas debidas al deterioro progresivo, dolor mal controlado, abandono del paciente crónico, encarnizamiento terapéutico y uso de medidas innecesarias que posponen la muerte. Se toman en un contexto complejo de dilemas religiosos, éticos y legales.

Palabras Clave: Enfermo terminal, toma de decisiones, dolor, derecho a morir, eutanasia, derechos del paciente (fuente: DeCS, BIREME). 


\begin{abstract}
Objectives Describing terminally-ill patients and their relatives' preferences regarding end of life decisions, the underlying problems and reasons leading to them requesting support.

Methods This was a descriptive, exploratory analysis of the secondary sources in an NGO providing support for patients at the end of their lives.

Results The most frequent choice was dying with dignity (43\%), followed by euthanasia (12\%). Some people asked for guidance on legal and ethical aspects regarding appropriate care for the terminally-ill. The pathologies accounting for $75 \%$ of the diagnoses were chronic degenerative diseases, cancer and coma. The worsening of symptoms was the main reason for requesting support and uncontrollable pain more often led to the choice of euthanasia. Only $14 \%$ of the patients had formalised their wills regarding their end of life decisions. The choice of dying with dignity was related to rejecting futile measures and therapeutic cruelty. Euthanasia was seen as an autonomous intentional action to end suffering. The family plays an essential role in making end of life decisions.

Conclusions End of life decisions are motivated by a perception of undignified conditions for patients due to progressive deterioration, poorly controlled pain, abandoning of chronic patients, therapeutic obstinacy and unnecessary measures that postpone death. They are taken within a complex context concerning religious dilemmas and ethical or legal concerns.
\end{abstract}

Key Words: Terminally ill, decision-making, pain, right to die, euthanasia, patient's rights (source: $M e S H, N L M$ ).

a muerte ha sido por lo general un tema que se considera negativo y se excluye de las conversaciones cotidianas. Sin embargo, cuando se tiene una edad avanzada o una enfermedad grave, se piensa con frecuencia en ella y en las condiciones en que sucederá (1-3). El proceso de toma de decisiones depende no solo de las opciones reales sino de las opciones imaginadas, de la forma en que las personas han comprendido la información a la que han tenido acceso, de las creencias y del curso de la enfermedad (4). En este proceso, pacientes y familiares buscan indagar más sobre la patología y solicitan orientación por parte de conocidos o del personal de salud. Es una época de crisis y altos niveles de estrés, pues se trata justamente de decidir y reflexionar sobre asuntos trascendentales del ser humano como la vida y la muerte (5). Aun así, en la mayoría de las instituciones de salud no se tiene el tiempo, los recursos ni la disponibilidad para atender estas necesidades, tal como lo han demostrado reconocidos estudios como el SUPPORT realizado en 1995 (6). Aun así, se han diseñado en los últimos años algunas estrategias para respetar la autonomía de los 
pacientes en la toma de decisiones como el Consentimiento Informado, el Código de No Reanimación y el manifiesto de las Voluntades Anticipadas. En algunos países el respeto de la autonomía incluye la posibilidad de la eutanasia o de suicidio asistido (7).

En el conjunto de opciones que se tienen en cuenta al final de la vida se utilizan diversas expresiones de manera ambigua debido particularmente al juicio moral de que son objeto ya sea como acciones u omisiones. Estas son, por ejemplo, eutanasia activa, eutanasia pasiva, homicidio por piedad, ayuda a morir, muerte digna, buen morir, distanasia, ensañamiento terapéutico, limitación del esfuerzo, vida artificial, cuidado paliativo y muerte natural, entre otros.

En el mundo actual, la atención de pacientes en fase avanzada de su enfermedad es deficiente, con pocas excepciones como El Reino Unido, Nueva Zelandia y Australia (8). En muchos casos la atención resulta en intervenciones que no representan mayor beneficio para los pacientes ni para sus familiares y devienen en onerosos gastos para los sistemas de salud (9). Ante las dificultades para que los enfermos terminales sean atendidos por las instituciones de salud, las familias o los voluntarios de la comunidad asumen su cuidado, aunque solo en algunos países desarrollados tienen un reconocimiento social o económico (10).

Los pacientes que padecen enfermedades en fase avanzada presentan diversos síntomas, entre los que prevalece el dolor (11). Disnea, anorexia, vómito, sangrados y alteraciones en la orina y la deposición causan sufrimiento al paciente, quien ya se encuentra al límite de su tolerancia debido a su grave enfermedad. Este sufrimiento se extiende también a los cuidadores, quienes deben hacer un gran esfuerzo físico y emocional para manejar los pacientes (12).

Expertos de la Organización Mundial de la Salud, afirman que el dolor es controlable entre un 80 y un $90 \%$ de los casos con un esquema ascendente de analgésicos opioides (13). Actualmente hay medicamentos disponibles en el mercado con alta potencia y suficiente variedad para poder combinar o intercambiar su uso como Fentanilo, Metadona, Morfina, Oxicodona o Petidina. No obstante, algunos clínicos, particularmente oncólogos, opinan que el dolor sigue siendo uno de los problemas más frecuentes y de difícil manejo (14). 
Varios especialistas han expuesto las razones por las cuales el dolor no está siendo bien manejado y se encuentra sub-tratado: Los médicos no reciben una educación suficiente para el manejo del dolor; están más concentrados en controlar la enfermedad que en manejar sus síntomas; temen a la adicción de los pacientes o a las sanciones que eventualmente puedan tener por formular estos medicamentos; los pacientes no lo reportan o rechazan el uso de opiáceos por la creencia de que solo se usan cuando ya la muerte es inminente (15-18).

En Colombia no existe una política explícita de atención en salud para el final de la vida (19). Hay muy pocos especialistas en cuidado paliativo, y, aunque algunas instituciones ofrecen programas de atención domiciliaria, no cumplen con los criterios de una atención completa en cuidado paliativo (20). El uso de morfina para el manejo del dolor es demasiado bajo, pues es inferior al promedio para América Latina y éste último corresponde a menos del $3 \%$ del consumo mundial, lo cual es un indicador de pobre oferta de cuidado paliativo (21).

Desde el punto de vista legal, Colombia es el único país latinoamericano en el cual se encuentra despenalizada la eutanasia en el caso de los enfermos terminales en que concurra la voluntad libre del sujeto (22), pero, esta sentencia de la Corte Constitucional no se ha reglamentado por el Congreso de la República, lo cual crea un ambiente legal incierto.

La Resolución 13437 de 1991 del Ministerio de Salud que reglamenta los derechos de los pacientes, toca tres aspectos referentes a la atención al final de vida: a. Las decisiones en caso de inconsciencia o minoría de edad deben ser tomadas por los familiares o representantes, dejando expresa constancia ojalá escrita de su decisión; b. En caso de enfermedad irreversible se deben respetar los deseos del paciente; c. El paciente tiene derecho a morir con dignidad y a que se le respete su voluntad de permitir que el proceso de la muerte siga su curso natural en la fase terminal de su enfermedad (23).

En este contexto existe en Colombia una ONG que apoya a personas que desean tener algún control sobre la forma y las circunstancias de su muerte. Para este trabajo se consultaron sus archivos y se analizó la información sobre el tipo de consultas y solicitudes que se realizaron durante 4 años y medio en su sede en la ciudad de Bogotá. El interés fue observar el tipo de 
decisiones que se presentan, las dificultades de los pacientes y familiares y los problemas que subyacen a las demandas de ayuda.

\section{MATERIALES Y MÉTODOS}

Estudio descriptivo, exploratorio. Se tomó información secundaria obtenida de los archivos físicos de la institución, con reserva de la identidad de las personas y manteniendo la confidencialidad. Se digitó, depuró y tabuló la información de la totalidad de registros para este período $(\mathrm{N}=513)$ desde el año 2004 hasta mediados del año 2008, utilizando el programa Excel para Windows 7®.

\section{RESULTADOS}

Las características de 513 pacientes registrados fueron las siguientes: El género fue femenino en un 53,6 \% y el grupo de edad más frecuente fue el de mayores de 60 años (66 \%) (Tabla 1). Tres diagnósticos correspondieron al 75 \% de los casos: La Enfermedad Crónica Degenerativa, el Cáncer y el "Estado de Coma”. Bajo la categoría de Enfermedad Crónica Degenerativa se encontraron Esclerosis Lateral Amiotrófica, Esclerosis Múltiple, Parkinson, Alzheimer, Diabetes Mellitus, Insuficiencia Renal Crónica, Enfermedades Pulmonares Obstructivas, Osteoporosis y Enfermedades del Colágeno (Tabla 2).

Tabla 1. Distribución por grupos etarios de 513 pacientes que consultaron a ONG de apoyo a las personas al final de la vida Bogotá, Colombia. 2004 -2008

\begin{tabular}{lcc}
\hline Grupo etáreo & Número & $\%$ \\
\hline $0-14$ & 22 & 4,3 \\
$15-44$ & 37 & 7,2 \\
$45-59$ & 48 & 9,4 \\
\hline $60-69$ & 37 & 7,2 \\
$70-79$ & 75 & 14,6 \\
\hline 80 y más & 100 & 19,5 \\
Sin información & 194 & 37,8 \\
\hline Total & 513 & 100 \\
\hline
\end{tabular}


Tabla 2. Diagnóstico de los pacientes en 513 consultas realizadas a una ONG de apoyo a las personas al final de la vida. Bogotá, Colombia $2004-2008$

\begin{tabular}{lcc}
\hline Diagnóstico & Número & $\%$ \\
\hline Enfermedad crónica degenerativa & 168 & 32.8 \\
\hline Cáncer & 143 & 27,9 \\
Estado de coma & 72 & 14,0 \\
\hline Otras & 98 & 19,0 \\
Accidente cerebro-vascular & 17 & 3,4 \\
Daño cerebral & 15 & 2,9 \\
\hline Total & 513 & 100 \\
\hline
\end{tabular}

En la Tabla 3 y el Cuadro 1 se describen los motivos de consulta de todos los pacientes, siendo el más frecuente la Muerte Digna con un 43 \%. Los pacientes o sus familiares relacionaron la indignidad de la muerte con varias situaciones, pero las más frecuentes fueron el dolor intolerable, la mala calidad de vida debido a la incapacidad física, el deterioro progresivo y el permanecer en estado de inconsciencia por tiempo prolongado.

"ILa vida para ella es todo un tormento! Tiene dolor siempre. A veces más y otras veces mucho más! En la noche duerme, si mucho una hora; el resto se la pasa de un lado a otro buscando una posición soportable. Si se le dice algo, grita, regaña y maldice. A veces duerme un poco en la mañana." Cónyuge de una paciente con cáncer terminal 1:84 (30:30).

Tabla 3. Motivos de consulta de 513 personas que solicitaron ayuda a una ONG de apoyo a las personas al final de la vida. Bogotá,

$$
\text { Colombia } 2004-2008
$$

\begin{tabular}{lcc}
\hline Motivo de consulta & Número & $\%$ \\
\hline Muerte Digna & 222 & 43,3 \\
\hline Orientación & 114 & 22,2 \\
Eutanasia & 60 & 11,7 \\
Otros & 45 & 8,8 \\
Cómo hacer para que respeten su voluntad & 20 & 3,9 \\
Dolor insoportable & 19 & 3,7 \\
Cómo hacer para que no le prolonguen la vida & 14 & 2,7 \\
Conflicto familiar & 11 & 2,1 \\
Mala calidad atención en salud & 8 & 1,6 \\
\hline Total & 513 & 100 \\
\hline
\end{tabular}


Cuadro 1. Motivos de consulta según los principales diagnósticos de 513 personas que solicitaron ayuda a una ONG de apoyo a las personas al final de la vida. Bogotá, Colombia 2004 -2008

\begin{tabular}{|c|c|c|c|c|c|}
\hline No. & ACV & Cáncer Terminal & $\begin{array}{c}\text { Cáncer no } \\
\text { Terminal } \\
\end{array}$ & Coma & $\begin{array}{c}\text { Enf. Crónica } \\
\text { Degenerativa }\end{array}$ \\
\hline $1^{\circ}$ & $\begin{array}{c}\text { No mas } \\
\text { intervenciones }\end{array}$ & Muerte Digna & Orientación & Muerte digna & Muerte digna \\
\hline $2^{\circ}$ & Orientación & Eutanasia & Muerte Digna & Orientación & Eutanasia \\
\hline $3^{\circ}$ & $\begin{array}{l}\text { Respetar } \\
\text { voluntad } \\
\text { paciente }\end{array}$ & $\begin{array}{l}\text { No mas } \\
\text { intervenciones }\end{array}$ & Temores & $\begin{array}{l}\text { Conflicto } \\
\text { familiar por } \\
\text { decisiones }\end{array}$ & Dolor \\
\hline $4^{\circ}$ & Orientación & Orientación & Eutanasia & Orientación & Orientación \\
\hline $5^{\circ}$ & Eutanasia & Dolor & $\begin{array}{c}\text { No mas } \\
\text { intervenciones }\end{array}$ & Eutanasia & Otros \\
\hline
\end{tabular}

Las personas hacen una diferencia cuando solicitan muerte digna o eutanasia, optando la mayoría por la primera como una elección más conservadora, prudente y acorde con sus creencias religiosas y morales. Se encontró en este grupo de personas que la idea de muerte digna se relaciona con la idea de una muerte tranquila, sin dolor, sin asfixia, sin angustia y "sin máquinas" (respirador, monitores, tubos, catéteres o sondas). Desde el punto de vista psicológico, con una sensación de paz, de haber resuelto sus preocupaciones, de haberse podido despedir de las personas queridas y de haber podido hacer sus rituales religiosos. El lugar preferido es la casa, pero no quieren sentirse abandonados por el personal de salud.

"Mi madre quiere que no la molesten más, no más terapias, ni mas clínica, que pueda estar relajada y morir tranquila." Hija de una señora de 73 años con diabetes fase terminal $1: 24(156: 156)$

Algunos solicitaron orientación relacionada principalmente con los siguientes temas: cómo tener una muerte digna; cómo pueden ayudarle a morir; cómo pueden darle una mejor atención a su familiar en la casa, cómo calmarle el dolor y atender otros síntomas; dónde llevar a un paciente inconsciente cuando se lo entregan en el hospital. También quieren saber si algunas conductas o decisiones particulares con los pacientes son éticas o permitidas por la ley; si están actuando correctamente en determinada circunstancia o cómo pueden comunicarse con los médicos para expresar sus temores y deseos. Los pacientes y familiares tienen miedo a hablar con los médicos y enfermeras debido al rechazo, maltrato o incomprensión. También tienen miedo a que no se respete su voluntad y al uso de medidas innecesarias por desconocimiento del pronóstico, la edad o las condiciones del paciente. 
"Quiero me que digan cómo hablar con los médicos para que lo desconecten. Ellos dicen que desconectarlo es anti-ético." Hermana de un hombre de 42 años en coma profundo hace 10 meses - 1:66(65:65)

La solicitud explícita de ayuda para provocar la muerte, se clasificó como Eutanasia. Se presentó en el 12 \% de las consultas y se manifestó en pacientes que sufrían dolor o asfixia crónica progresiva que consideraban insoportable, deterioro severo de sus capacidades físicas, compromiso de múltiples sistemas del organismo o un pronóstico a mediano plazo de deterioro mental o invalidez progresiva irreversibles.

Los motivos de consulta variaban según el diagnóstico o según la fase de la enfermedad del paciente. Probablemente la agudización de los síntomas hacia la etapa final de patologías como el cáncer cambia las prioridades y los pacientes contemplan opciones mucho más concretas, pasando de la solicitud de información a la solicitud de apoyo para evitar más intervenciones o para acelerar la muerte. Con todo, la muerte digna es la opción más solicitada.

"Queremos ayudarle a morir dignamente. Queremos que le calmen el dolor, que le den la morfina en la dosis que necesita y durante todo el tiempo para que esté tranquilo". Familiar de un paciente con cáncer terminal - 1:24(9:9)

En relación con las patologías de los pacientes, el diagnóstico más frecuente fue la Esclerosis Lateral Amiotrófica. Los pacientes que la padecían, así como sus familiares expresaban como síntomas más agobiantes el deterioro físico progresivo y la pérdida de sus capacidades. Se sentían exhaustos y eran conscientes de la carga que representaba su cuidado para sus familiares.

"El paciente viene personalmente y habla de su situación de dependencia, del avance de la enfermedad, de que no puede hacer las cosas que antes hacía... Tiene cada vez más dificultad para comer, tiene muchas flemas. Necesita a alguien que le ayude permanentemente. Expresa su voluntad de que le sea aplicada la eutanasia en el momento que él considere que ya no tiene sentido seguir viviendo, por la indignidad de su condición." Nota en el archivo de un paciente de 54 años con Esclerosis Lateral Amiotrófica 1.1 (29:29)

El segundo diagnóstico fue el Cáncer terminal y su frecuencia fue mayor en el grupo que solicitaba eutanasia. El tercero fue el “Coma” . En este caso, los familiares argumentaban la mala calidad de vida, las complicaciones 
infecciosas, la necesidad de utilizar equipos para aspiración de tráquea o sonda de gastrostomía, las escaras, y el pronóstico negativo.

"Queremos que nuestra madre tenga un buen morir. Lleva cuatro años deteriorándose por un derrame. Está entumida, no puede hablar, no puede comer, no camina y tiene escaras que llegan hasta el hueso, mucho dolor. Cada día está peor." Hija de una paciente de 81 años - 1:94 (102:102)

Más de la mitad de las consultas fueron realizadas por una hija (o) del paciente, o por un hermano (a), mientras que el paciente consultó personalmente solo en el $10 \%$, como se observa en la Tabla 4. Esto muestra que, para nuestra cultura, la familia tiene un papel muy importante en el acompañamiento a los enfermos al final de la vida y en la toma de decisiones. Consistente con esta información, se hizo evidente también la poca costumbre de manifestar por escrito las Voluntades Anticipadas, las cuales fueron expresadas formalmente solo en el $14 \%$ de los pacientes. Por este motivo, son los hijos quienes asumen con muy alta frecuencia la representación de sus padres enfermos, tanto para solicitar orientación, como para solicitar la eutanasia o la muerte digna. Aún así, esta representación en la mayoría no está formalizada en ningún documento.

Tabla 4. Parentesco en relación con el paciente de 513 personas que solicitaron ayuda a una

ONG de apoyo a las personas al final de la vida. Bogotá, Colombia 2004 -2008

\begin{tabular}{lcc}
\hline \multicolumn{1}{c}{ Parentesco } & Número & $\%$ \\
\hline Hijo & 228 & 44,4 \\
Paciente & 53 & 10,3 \\
Hermano & 51 & 9,9 \\
Cónyuge & 23 & 4,5 \\
Otros & 139 & 26 \\
\hline Sin información & 19 & 3,7 \\
\hline Total & 513 & 100 \\
\hline
\end{tabular}

El $37 \%$ de las solicitudes de eutanasia fueron realizadas por el paciente de a sus familiares o a la ONG de manera directa, como se observa en afirmaciones como las siguientes:

"Mi padre nos pide que le ayudemos a morir! Está buscando un arma para matarse." Familiar de un paciente de 71 años con cáncer terminal 1:31(60:60) 
"Mi padre está desesperado. Nos dice que le amarremos una bolsa plástica en su cabeza para que pueda morir ya. Por eso vinimos a buscar ayuda." Hija de un paciente con Esclerosis Lateral Amiotrófica 1:63 (25:37).

\section{DISCUSIÓN}

Los resultados de este estudio deben comprenderse en el contexto de un grupo de personas que se encuentran al final de la vida y que son diferentes a la mayoría de los pacientes por tres razones: 1. Piden apoyo; 2. Quieren control sobre el proceso y; 3. Saben que hay una institución que les puede brindar ayuda. En este sentido, las inferencias a otros grupos deben ser prudentes. Es posible que haya personas con similares situaciones en las casas o en los hospitales, pero se desconoce qué piensan o cómo han actuado.

En este trabajo se encontró que la solicitud de ayuda al final de la vida se motivó por cinco situaciones claras: a. Dolor incontrolable; b. Deterioro progresivo; c. Incapacidad física; d. Estado de inconsciencia prolongado y; e. Violación de los derechos de los pacientes.

Como lo afirman otros investigadores $(24,25)$, se observó que el sufrimiento al final de la vida es intenso y es debido en muy gran medida al dolor. En este trabajo se comprobó que este síntoma produce un deterioro significativo de la calidad de vida y es motivo de depresión, agotamiento físico y psicológico, aislamiento del paciente y de los familiares. Teniendo en cuenta que existen medios terapéuticos para controlarlo, es ineludible que en Colombia se defina una política clara y contundente de cuidado paliativo que incluya entre otros servicios el uso de opioides en dosis adecuadas y correspondientes a la demanda real de la población.

En este estudio se encontró también que el dolor intolerable motiva con mucha frecuencia la toma de decisiones radicales como la eutanasia, por lo cual, en todos los pacientes que la solicitan, debe ser un requisito haber intentado controlarlo de manera satisfactoria para evaluar posteriormente dicha solicitud.

Del análisis de las consultas y demandas de ayuda se concluye que cuando los pacientes solicitan muerte digna están pidiendo que les controlen el dolor, los acompañen y les respeten su proceso de morir tranquilamente, 
en un ambiente íntimo, sin intromisiones ni dispositivos tecnológicos que pospongan la muerte. Aunque debe explorarse más profundamente a través de fuentes primarias, parece ser que, para las personas que conformaron el grupo de estudio, el concepto de muerte digna es diferente al de eutanasia, particularmente por su valoración moral. La muerte digna se asume como una muerte natural, mientras que la eutanasia se entiende como la acción intencional para producir la muerte de un enfermo terminal. Esta diferencia no se encuentra tan marcada en otros países. Por ejemplo, en España, las personas a favor de la eutanasia se apoyan en el derecho a una muerte digna y parece que los dos términos se utilizan como sinónimos (26), mientras en Suiza o Estados Unidos morir dignamente es sinónimo de suicidio asistido (27).

Otro aspecto que se destaca en este estudio es la percepción de una actitud distante de los médicos que genera temor, desinformación y ansiedad. Además, los pacientes perciben que el médico pierde todo interés cuando ellos son declarados enfermos "terminales”, originando la sensación de abandono, lo cual coincide con las conclusiones de otros estudios publicados (28). Los pacientes y familiares consideran que no se están respetando los derechos de los pacientes porque se impide que la muerte siga su curso natural como resultado de una enfermedad crónica interviniendo para posponerla y porque se actúa en contra de la voluntad expresa de los pacientes o responsables.

Posteriores estudios deben ser complementados con información obtenida directamente con los pacientes y familiares para profundizar en posibles explicaciones de los hallazgos de este trabajo exploratorio

\section{REFERENCIAS}

1. Lloyd-Williams M. The end of life: a qualitative study of the perceptions of people over the age of 80 on issues surrounding death and dying. J Pain Symptom Manage. 2007; 34(1):60-6.

2. Givens J, Mitchell S. Concerns about end-of-life care and support for eutanasia. J Pain Syntom Manage. 2009; 38(2):167-73.

3. Murray S, Boyd K, Kendall M, Worth A, Benton F, Clausen H. Dying of lung cancer or cardiac failure: prospective qualitative interview study of patients and their carers in the community. BMJ. 2002; 325(7370):929.

4. Neimeyer R. Death Anxiety Handbook. Washington: Neimeyer Robert Editor; 1994. p. 13 -16.

5. Block S. Psychological Considerations, Growth and Transcendence at the End of Life. JAMA. 2001; 285:2898-2905. 
6. The Support Group. A controlled trial to improve care for seriously ill hospitalized patients (The Study to Understand Prognoses and Preferences for Outcomes and Risks of Treatment). Jama. 1995;274:1591-1598.

7. ProCon.org [Internet]. International Perspectives Legal Status of Euthanasia and Assisted Suicide. Disponible http://euthanasia.procon.org/view.resource.php?resourcelD=000136 Consultado: Enero 20 de 2012.

8. The Economist Intelligence Unit [Internet]. The quality of death: ranking end-of-life care across the world. 2010 p 12. Disponible: http://www.eiu.com/site_info.asp?info_name=qualityofdeath lienfoundation\&page=noads\& $\mathrm{rf}=0$ Consultado: Enero 22 de 2012.

9. The CBS News [Internet].. The cost of dying: end-of-life care. Patient's last two months of life cost medicare $\$ 50$ billion last year; Is there a better way? Agosto 8 de 2010 . Disponible: http://www.cbsnews.com/stories/2010/08/05/60minutes/main6747002. shtml. Consultado: Enero 22 de 2012.

10. Rodríguez G [Internet]. Protección de la dependencia en España. Fundación Alternativas. 2004: 31-39. Disponible: http://mayores.pre.cti.csic.es/documentos/documentos/ rodriguez-proteccion-02.pdf. Consultado: Enero 20 de 2012.

11. U.S. National Institutes of Health-National Cancer Institute [Internet]. Last Day of Life. Symptoms Management. Health Professional Section. Disponible: http://www.cancer.gov/cancertopics/pdq/supportivecare/lasthours/healthprofessional/ page1 Consultado Enero 18 de 2012.

12. Institute of Medicine Committee on Care at the End of Life [Internet]. Approaching Death: Improving Care at the End of Life -Washington DC .National Academy Press. 1997: 40. Disponible: http://www.nap.edu/openbook.php?record_id=5801. Consultado: Enero 23 de 2012.

13. Van den Beuken, Van Everdingen M, De Rijke J, Kessels A, Schouten $\mathrm{H}$, van Kleef M y Patijn J. Prevalence of pain in patients with cancer: a systematic review of the past 40 years. Ann Oncol. 2007; 18:1437-1449.

14. Callahan D. Death and the research imperative. New England Journal of Medicine 2000; 342 (9): 654-656.

15. Rousseau P. The neglect of pain: the sad tale of opioids at the end of life. Am J Hosp Palliat Care. 2010;27: 97-98.

16. Bloodworth D. Opiods in the treatment of chronic pain: legal framework and therapeutic indications and limitations. Phys Med Rehabil Clin N Am. 2006; 17: 355-79.

17. Kahan M, Srivastava A, Wilson L, Gourlay D, Midmer D. Misuse of and dependence of opioids: study of chornic pain patients. Can Fam Physician. 2006; 52:1081-7.

18. Colombia [Comisión de regulación en salud] [Internet]. Acuerdo No.08 de 20 Diciembre de 2008. Disponible: http://www.pos.gov.co/Documents/Acuerdo\%20008\%20Dic\%202909.pdf Consultado: Enero 20 de 2012.

19. Palliative Care. In: World Health Organization [Internet]. Cancer Control: Knowledge into action. W.H.O. guide to effective programmes Module 5. Ginebra: World Health Organization; 2007. Disponible: http://whqlibdoc.who.int/publications/2007/9789241547345_eng.pdf. Consultado: Enero 12 de 2012.

20. León M, De Lima L, Flórez S, Torres M, Daza M, Mendoza L, Agudelo N, Guerra L y Ryan K. Improving availability of and access to opioids in Colombia: description and preliminary results of an action plan for the country. Journal of Pain and Symptom Management 2009; 38(5):758-766.

21. Colombia Corte Constitucional [Internet]. Sentencia C-239/97. Disponible: http:// www.procuraduria.gov.co/guiamp/Macroproceso\%20Disciplinario/ Providencias/97-C-239\%20Eutanasia.htm Consultado: Enero 20 de 2012 
22. Colombia [Ministerio de Salud] [Internet]. Resolución 13437 de 1991 por la cual se constituyen los comités de ética hospitalaria y se adopta el decálogo de los derechos de los pacientes. Artículos $2^{\circ}, 5^{\circ}$ y $10^{\circ}$. Disponible: http://webcache.googleusercontent.com/ search?q=cache:kJAL7ILAX-8J:www.medicosgeneralescolombianos.com/RESOLUCI ON\%252013437\%2520DE\%25201991\%2520derechos\%2520de\%2520los\%2520pa cientes.doc+Resoluci\%C3\%B3n+n\%C3\%BAmero+13437+de+1991+(Ministerio+de+ Salud+P\%C3\%BAblica),\&cd=3\&hl=es\&ct=clnk\&gl=co Consultado: Enero 22 de 2012.

23. Jacobsen R, Liubarskienë Z , Idrup C, Christrup L, Sjgren P, Samsanavièienë S. Barriers to cancer pain management: a review of empirical research. Medicina (Kaunas) 2009; 45(6) :427-433. Disponible: http://medicina.kmu.lt/0906/0906-01e.pdf. Consultado: Enero 13 de 2012.

24. Rousseau P. The neglect of pain: the sad tale of opioids at the end of life. Am J Hosp Palliat Care. 2010;27: 97-98.

25. Asociación Federal Derecho a Morir Dignamente en España [Internet]. Disponible: http:// www.eutanasia.ws/ . Consultado: Enero 13 de 2012.

26. Death With Dignity National Center [Internet]. Disponible: http://archive.deathwithdignity.org/ news/news/bostonglobe.02.27.06.asp Consultado: Enero 23 de 2012.

27. Back A, Young J, McCown E, Engelberg R, Vig E, Reinke L, Wenrich M, Mc Grath B \& Curtis Ret. Abandonment at the end of life from patient, caregiver, nurse, and physician perspectives: loss of continuity and lack of closure. Arch Intern Med. 2009; 169(5):474479. 